SciDioc

\section{Evaluation of Apically Positioned flap with A_PRF Vs. Free Gingival Grafts to Enhance the Keratinized Tissue Around Dental Implants: A Randomized Controlled Clinical Split Mouth Trial}

Research Article

Jihad ALsahli ${ }^{1}$, Tarek Kasem², Muaaz Alkhouli ${ }^{3 *}$

${ }^{1}$ Department of Periodontology, Faculty of Dentistry, Damascus University, Syria.

${ }^{2}$ Professor at Department of Periodontology, Faculty of Dentistry, Damascus University, Syria.

${ }^{3}$ Department of Pediatric Dentistry, Faculty of Dentistry, Damascus University, Syria.

\title{
Abstract
}

\begin{abstract}
Aim: The essential goal of mucogingival plastic surgery is to obtain an adequate keratinized tissue and an optimal gingival phenotype, The aim of this study was to evaluate the efficacy of Advanced platelet-rich fibrin (A-PRF) with Apically Positioned flap (APF) compared to Free Gingival Grafts (FGG) with APF during dental implants second surgical.

Material and Methods: In this study, 30 Periodontal Plastic-Surgery were performed for 15 patients in the posterior area of the upper/lower jaws in a split mouth, Where one of the sides was treated (randomly) by APF with A-PRF //Test group// and the other sides treated with FGG //Control group//. - Clinical parameters were taken and included: Width of keratinized tissue (WKT), Gingival thickness (G.T), healing index (HI), pain index (PI) in different periods of time.

Results: At 2 months of healing (T2), the mean (SD) width of the keratinized tissue was $8.54 \pm 0.75$ and $8.63 \pm 1.18 \mathrm{~mm}$ for the test group and control group respectively $(\mathrm{P}>0.05)$. at 6 months of healing (T6), the mean (SD) thickness of the keratinized tissue (Phenotype) was $2.87+0.51$ and $2.86 \pm 0.53 \mathrm{~mm}$ for the test group and control group respectively $(\mathrm{P}>0.05)$.and results showed a noticeable improvement in the pain amount values and the degree of healing in the test group $(\mathrm{P}<0.05)$. Conclusions: Within the limits of this study, the A-PRF group provided better healing and less pain than the control group and was effective treatment option and could serve as an alternative to FGGs. Clinical significance: Patient-related outcomes are of utmost importance in clinical practice. The use of (A-PRF\&APF) provides an inexpensive and reliable alternative to improve postoperative pain and healing in mucogingival plastic surgery.
\end{abstract}

Keywords: Advanced Platelet Rich Fibrin; Apically Positioned Flap; Dental Implants; Free Gingival Grafts; Keratinized Tissue.

\section{Introduction}

Numerous examinations showed that the hard tissue experiences dimensional shrinkage following an extraction, while the keratinized tissue (KT) zone and vestibular depth in the soft tissue are both reduced to differing degrees [1]. These alterations are more prominent in cases where the extraction is indicated due to periodontitis diseases. The diminished height of the alveolar process, due to crestal bone resorption, brings about the loss of the KT because of the decreased distance between the bone crest and the genetically defined position of the mucogingival line [2].

Thanks to the appearance of dental implants, the structure and function of the mucosa that encompasses an implant have been thoroughly studied, since the presence of healthy peri-implant soft tissues is needed to provide an ideal seal between the oral environment and the implant with its superstructure, which plays a crucial role in the long-term success of a dental implant.

The width of the KT varies between different individuals, and between the teeth of the same individual. The minimum width of KT essential for maintaining periodontal health and counteracting gingival recession has not yet been determined; and this subject remains a matter of discussion. However, The absence of an adequately wide zone of $\mathrm{KT}$ is suspected to be a potential contributing variable for peri-implant disease. Some studies found no relation between the absence of $\mathrm{KT}$ and the quality of

*Corresponding Author:

Muaaz Alkhouli MSc, DDS,

Department of Pediatric Dentistry, Faculty of Dentistry, Damascus University, Syria.

Email ID: Muaaz.alkhouli@outlook.com

Received: January 22, 2021

Accepted: February 28, 202

Published: March 03, 2021

Citation: Jihad ALsahli, Tarek Kasem, Muaaz Alkhouli. Evaluation of Apically Positioned flap with A_PRF Vs. Free Gingival Grafts to Enhance the Keratinized Tissue Around Dental Implants: A Randomized Controlled Clinical Split Mouth Trial. Int J Dentistry Oral Sci. 2021;08(03):1844-1850. doi: http://dx.doi.org/10.19070/2377-8075-21000366

Copyright: Muaaz Alkhouli 2021 . This is an open-access article distributed under the terms of the Creative Commons Attribution License, which permits unrestricted use, distribution and reproduction in any medium, provided the original author and source are credited. 
plaque control, peri-implant health, and marginal bone loss [3], while others linked the lack of sufficient $\mathrm{KT}$ width with increased plaque accumulation, a higher gingival index, expanded probing depth, increased bleeding on probing, greater recession, and even marginal bone loss [4].

Many Research indicated that keratinized tissue around the abutments are play a significant role in peri implant health [5], and suggests that the presence of a sufficient amount of KT around to the implant decreases gingival inflammation, and hyperplasia, and limits marginal peri-implant gingival tissue recession.

Hence, soft tissue augmentation ought to be considered to provide an adequate keratinized tissue zone that envelops as wide an area of the implant prosthesis as possible, Soft tissue management in areas of implant restoration can be performed before or after the surgical phase, before the prosthetic stage, or after the consummation of the prosthetic phase. Various mucogingival surgical techniques have been developed for making or augmenting the KT around the teeth and implants; Including: Free gingival grafts (FGG), Lateral pedicle rotating flap, Apically positioned flap (APF), Coronally repositioned flap (CRF), Sub-epithelial connective tissue grafts (SCTG) and Cellular Dermal Grafts.

The APF has been found to increase the width of the KT around the teeth .This happens due to an apical change of the mucogingival junction, which involves an apical displacement of the muscular insertions [6]. The advantages of APF are minimal postoperative bone loss, control over the post-operative location of the gingival margin, and not requiring a second surgical site, which results in better patient acceptance and comfort [7]. Its disadvantages are that it can't be supported for a thin gingival Phenotype and the possibility to form a white scar. Today, the golden standard to increase the KT is a free gingival graft (FGG) from the palate [8]. However, the main reported issues following the FGG technique are agony and morbidity for the patient; as it includes a second surgical site, as well as a change in diet, paresthesia, herpetic injury, mucocele, arteriovenous shunt, and bleeding.

Efforts to overcome these disadvantages included attempts to supplant the autogenous tissue with an alternative material to reduce the patient's pain [9]. However, synthetic materials unfortunately come at a significant cost, and some biomaterials cannot supplant natural tissues.

Advanced-platelet-rich fibrin (A-PRF) is a third-generation platelet concentrate developed by Prof. Ghanaati [10]. It can be produced by a basic and modest method that doesn't require anti-coagulants. Its 3-dimensional fibrin network stimulates neovascularization, quickens wound healing, and accelerates tissue remodeling [11]. Platelet concentrates are viewed as a source of autologous development factors that stimulates cell movement and proliferation. Given that A-PRF is created without utilizing any added substance, the fibrin polymerization happens in a physiological way, bringing about a fibrin network comparable to networks shaped during natural healing.

A-PRF has been used in various surgical settings, including periodontology, implant dentistry and maxillofacial surgery [12]. In oral medical procedures, A-PRF was linked to lower postoperative pain and discomfort [13].
The aim of study was to evaluate of the use ofAdvanced plateletrich fibrin(A-PRF) Vs. Free Gingival Grafts (FGG) during dental implants second surgical phase in causing positive changes in the width of keratinized gingiva and in gingival Phenotype and assess the pain sensation and healing process.

\section{Materials and Methods}

\section{Study Design and Participants}

This study is a randomized controlled clinical trial with a split mouth design. The study included 15 patients $(7$ males and $8 \mathrm{fe}-$ males) who had reported to the faculty of dentistry in Damascus university in Syria, with ages between 22 and 65 years (mean 44.3 \pm 8.5 ); all patients were diagnosed with partially edentulous dentitions and required a replacement for missing teeth in the posterior region of the mandible and the maxilla.

The sample size was determined based on the null hypothesis, which states that the test group (APF+A-PRF) and the control group (FGG) weren't equal. The confidence level was determined by $95 \%$, the desired sample power was $95 \%$, then $G$ power (version 3.1.9) was used, and the required sample size was 13 patients (26 subjects). The selected study sample was increased by two persons to account for potential dropout patients, with the possibility of dispensing results of some subjects, The sample size was 15 persons.

After the study was explained to the patients, all patients completed a health history questionnaire to ensure the absence of systemic or local conditions that could compromise the periodontal surgical procedures. The study was conducted in accordance with the Helsinki Declaration of 1975 , as revised in 2000, and was approved by the internal Ethical Committee of the Damascus University No. 3296/SM. A written informed consent was obtained from all subjects who participated in the research study. This randomized clinical split mouth trail was registered in ISRCTN Registry of Clinical Trials with registration number: ISRCTN33695429.

\section{Inclusion criteria:}

1. Older than 18 years old.

2. Less than $2 \mathrm{~mm}$ of $\mathrm{KT}$ at the buccal site from the ridge crest on bilateral implants.

3. Patients have a thick gingival phenotype (GT $\geq 2 \mathrm{~mm})[14]$.

4. Adequate oral hygiene (API $\leq 1)$ ( API= Approximal Plaque Index) and goodgeneral health.

\section{Exclusion criteria:}

1. Smokers and Alcoholics ( $\geq 10$ cigarettes).

2. Patients with systemic diseases that could interfere with the healing.

3. Patients undergoing bisphosphonate treatment and patients who previously received radiotherapy for the jaws.

4. Patients with moderate to severe periodontitis.

5. Pregnant women and breastfeeding mothers.

Between December 2018 and December 2019, 15 patients were in need of bilateral soft tissue augmentation. Patients were randomly 
assigned to a test group (A-PRF) or a control group (FGG) in a split mouth design, via a randomization table; by a computer-generated randomization list (SPSS v23.0). The treatment methods (15 for A-PRF test group / 15 for FGGs control group).

\section{Surgical procedures}

At the time of surgery, 2\% BETADINE (povidone iodine) was used for rinsing the oral cavity for 2 minutes. Following local anesthesia using lidocaine hydrochloride with 1:100,000 epinephrine, a $15 \mathrm{c}$ blade was used to raise a partial-thickness mucosal flap by sharp dissection to create a bed free of muscle attachments on the buccal aspects of the soft tissue, the buccal flap was apically displaced and stabilized with periosteal sutures using resorbable sutures (Vicryl 6.0, Ethicon ${ }^{\circledR}$ ), and the margin of the flap was fixed with interrupted sutures. Then the cover screw was removed and the healing abutments were placed. After that, the surgery proceeded as follows:

\section{For the control group, [Fig1]:}

1. Harvesting a 1 to $2 \mathrm{~mm}$ thick FGG from, depending on the amount of tissue available in each patient's palate, and according to the tinfoil template.

2. Further uncovering of the implants and placing healing abutments.

3. Positioning and fixating the FGG on the recipient bed with crossed mattress sutures and interrupted non-resorbable sutures
(Nylon 5.0, Ethilon $\left.{ }^{\circledR}\right)$.

\section{For the test group, [Fig2]:}

1. Preparation of A-PRF: A standard venipuncture was performed with a butterfly needle into $10 \mathrm{~mL}$ tubes without anticoagulant using vacuum plain glass tubes ( A-PRF: Jiangxi Fenglin Medical Technology Co. Ltd, Fengcheng, China). A-PRF clots and membranes were prepared as described by Ghanaati and Choukroun. The tubes were immediately centrifuged at $1500 \mathrm{rpm}(208 \mathrm{~g})$ for 14 minutes using a table centrifuge (EBA 200, Hettich, Germany). After centrifugation, each A-PRF clot was removed from the tube and separated from the red blood cell (RBC) fractions at the base with scissors. A-PRF clots were gently squeezed for 5 minutes in a sterile A-PRF metal box (gravity, no loading) (Figure 2).

2. Suturing A-PRF membranes together with resorbable sutures (Vicryl 6.0, Ethicon ${ }^{\circledR}$ ).

3. Positioning and fixating the A-PRF membranes using healing abutment (poncho technique) and resorbable sutures (Vicryl 6.0, Ethicon $\mathbb{R})$.

No periodontal dressing was used for either group, and routine postoperative instructions and medications were given. Patients were scheduled for postoperative follow up after 1 week, 4 weeks, 8 weeks, and 6 months after the surgery. Baseline clinical measurements were taken immediately after surgery, and again after

Figure 1. Apically positioned flap with free gingival graft (FGG).

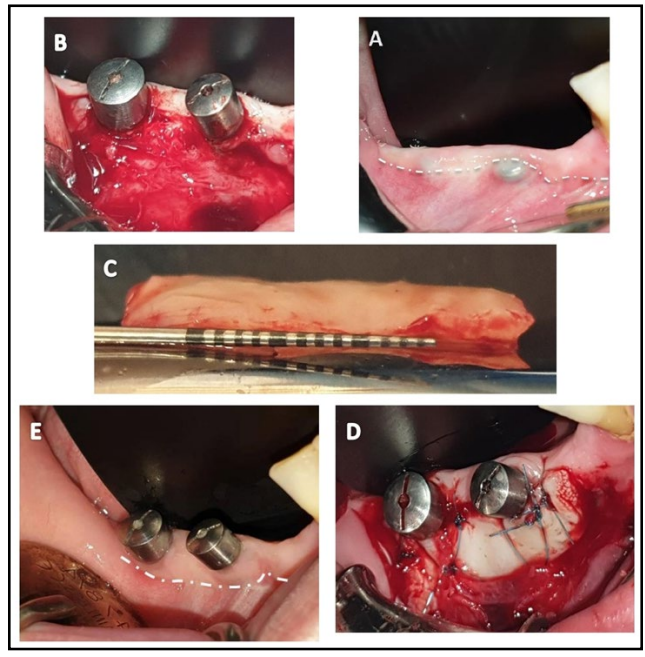

Figure 2. Apically positioned flap with Advanced platelet-rich fibrin (A-PRF).

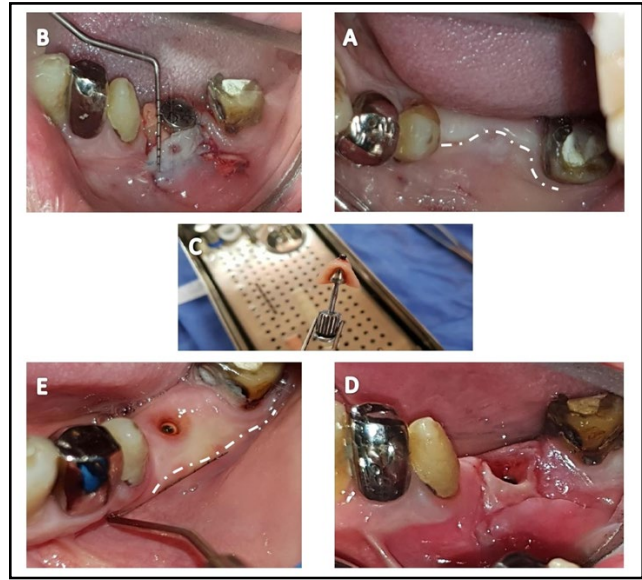


2 and 6 months after surgery. The width and thickness of the $\mathrm{KT}$ and the amount of graft shrinkage were the primary focus of the study. They were evaluated from the mucosal margin to the mucogingival junction, and measured at the center of each implant using a periodontal probe (UNC-15, Hu-Friedy Manufacturing Co., Chicago, IL) by using two special splints (Acrylic stent to measured thickness of keratinized tissue with periodontal probe and Vacuum stent to measured width of keratinized tissue with periodontal probe) measurements taking at the baseline, and again after 8 weeks, after performing apically displaced flap surgery [Fig 3 \& 4], and after 6 months. Gingival thickness measurements were taken using the acrylic stent, with an average of three measurements, as shown in [Fig 3]. Whereas, the width of the keratinized tissue was measured using the vaccum stent with periodontal probe from the lingual edge side of the stent to the mucogingival junction as shown in [Fig 4].

A secondary objective for the study was assessing healing and morbidity in both groups. A questionnaire was handed out to the patients and used $100 \mathrm{~mm}$ visual analog scores (VAS) to evaluate the amount of pain, ranging from 0 (no pain) to 100 (worst pain), and the patients were asked to fill the questionnaire in the VAS scales on the day of surgery every 4 hours (h), and afterwards daily until day 6 .

\section{Statistical Analysis}

The patient was considered a statistical unit for statistical analysis.
Statistical analyses were performed using a statistical package for social sciences program SPSS v23.0 (SPSS Inc, Chicago, IL, USA), $(\mathrm{P}<0.05)$ was considered Statistically significant for this study at $95 \%$ confidence interval,and we used Independent t-test, and Mann-Whitney $U$ to analyze the results.

\section{Results}

The study population consisted of 15 Patients with 30 bilaterally placed sides. The mean age was $41.6 \pm 10.6$ years Old and the male/female ratio was 8:6. One of the patients(male) dropped out During the 6 months follow-up. The two Contralateral implants in each patient Were assigned to the test group (A-PRF; 14 sides) or the control group (FGG; 14 sides).

One side in each patient was randomly assigned to either the test group (A-PRF; 14 sides) or the control group (FGG; 14 sides), while the contralateral implants was assigned to the other group.A flow diagram of the study participants is provided (Fig.6).

The healing process in the control group (FGG) and the test group (A-PRF) was uneventful. At baseline, both groups showed similar values for peri-implant health, plaque accumulation, gingival inflammation.

Before the surgery, the average total bucco-lingual width of the keratinized tissue was $6.28 \pm 0.22$ for the test group and $6.11 \pm$

Figure 3. Acrylic stent to measure thickness of keratinized tissue with periodontal probe.

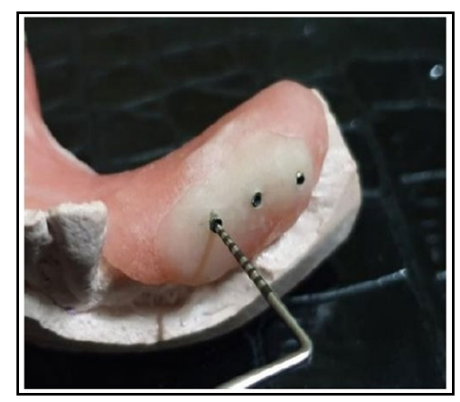

Figure 4. Vacuum stent to measured width of keratinized tissue with periodontal probe.

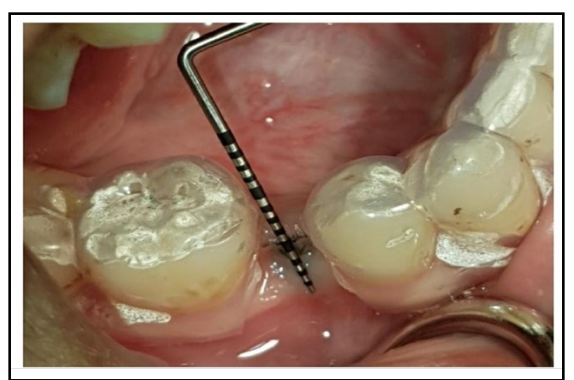

Figure 5. The mean of the visual pain values in the samples according to the time and the method of treatment : $*$ On the $\mathrm{X}$-axis, the time periods. *On the $\mathrm{Y}$-axis, the pain values.

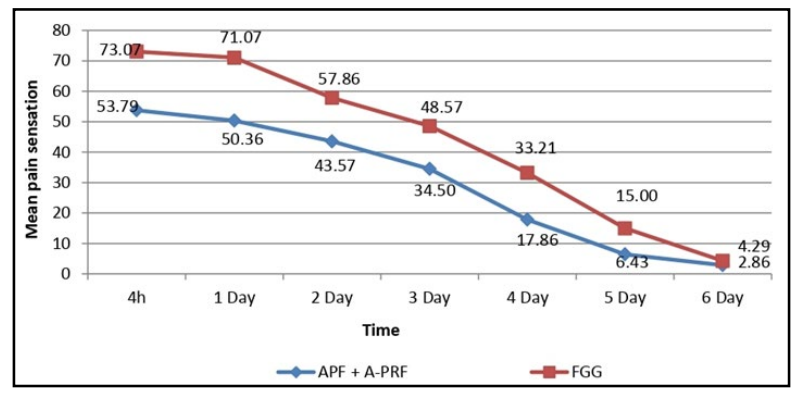


Figure 6. Flow diagram of the study.

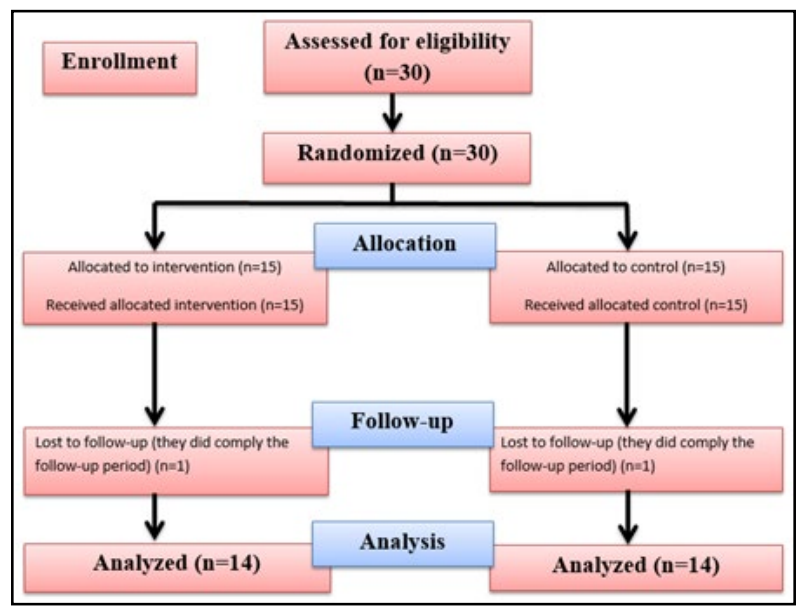

$0.22 \mathrm{~mm}$ for the control group, with no significant statistical difference between these values $(\mathrm{P}>.05)$. Immediately after the surgery (T0), the width of the KT formation was $10.51 \pm 0.21 \mathrm{~mm}$ for the test group and $10.81 \pm 1.30 \mathrm{~mm}$ for the control group, with no significant statistical difference between these values $(\mathrm{P}$ $>$.05). After 2 months of healing (T2), the width of the KT was $8.54 \pm 0.75$ and $8.63 \pm 1.18 \mathrm{~mm}$ for the A-PRF group and the FGG group respectively, and no significant statistical difference was noted between them $(\mathrm{P}>.05)$. With time, the total buccolingual width of the KT decreased. The mean gain of KT After 2 months of augmentation for both treatment methods demonstrated a significant increase in $\mathrm{KT}(\mathrm{P}<.05)$. [Table 1 ].

Shrinkage of the augmented siteswas measured by comparing the baseline postoperative buccal width of the KT (T0) with the width 2 months later (T2). The results were slightly different between both groups, with $20.02 \%$ for the test group and $18.56 \%$ for the control group. However, this difference was not statistically significant $(\mathrm{P}>.05)$. The relative width of the KT $(\%)$ at $\mathrm{T} 2$ exhibited a tendency to decrease when compared to its value at T0.

At baseline, the keratinized tissue thickness (Phenotype) was 2.35 \pm 0.49 and $2.38 \pm 0.56 \mathrm{~mm}$ for the A-PRF group and FGG group respectively, with no significant statistical difference between them ( $>$.05). After 2 months of healing (T2), the thickness of the KT was $3.44 \pm 0.49$ and $3.00 \pm 0.55 \mathrm{~mm}$ in the A-PRF group and the FGG group respectively, with a significant statistical difference between those values $(\mathrm{P}<.05)$. After 6 months of healing (T6), the thickness of the KT was $2.87+0.51$ and $2.86 \pm 0.53$ $\mathrm{mm}$ in the A-PRF group and the FGG group respectively, with no statistical difference between them $(\mathrm{P}>.05)$. With time, the thickness of the KT decreased in the test group, [Table 2 ].

Postoperative Pain Associated With A- PRF and FGGs Graft: This study evaluated patient pain sensation using a scale from 0 (absence of pain) to 100 (most severe pain). Each patient was asked to rate the values of pain score from day 1 until day 6 , and All the values from day 1 until day 5 were significantly higher in the control group $(\mathrm{P}<.05)$, [Fig 5 ].

\section{Discussion}

The periodontal health of dental implants is essential for provid- ing and maintaining functional and esthetic prostheses.

Many studies demonstrated that the presence of a certain width of keratinized tissue is important to maintain periodontal and peri-implant health, prevent soft tissue recession; protect the teeth and the implants from masticatory and external trauma, and to provide a barrier to inflammatory infiltrate [15].

Although a lack of keratinized tissue may not influence the longterm survival rate of implants [16], many studies have shown that the presence of a wide band of keratinized tissue is more important around restorations and prostheses than natural teeth [17], and leads to better oral hygiene results, better plaque control, and less gingival inflammation around implants [18]. Therefore, clinicians should consider it essential to provide and maintain adequate keratinized mucosa for the successful long-term maintenance of implants.

This study included only the posterior mandibular/Maxillae region with shallow vestibules and insufficient KT, after bone augmentation procedures could have led to shallow vestibules and a loss of buccal keratinized tissue. Even though such factors cannot be accurately quantified, they may generate high tension on the recipient site and accelerate shrinkage or relapse in the absence of a graft with mechanical resistance, (i.e., FGG). According to this definition, several research hasindicated that FGG was a Gold Standard and a better choice for sites exhibiting high atrophy and high muscle tension [19].

Numerous studies have shown that an increase in the amount of KT can be obtained by several different types of procedures, before, during or after implant placement [20].

Augmentation of the keratinized tissue width and vestibular deepening with autogenous FGGs has been reported to be a predictable and effective method [21]. But Even though FGG could effectively prevent wound contracture [22], it resulted in high morbidity, esthetical issues, increased surgical time and cost, and increased patient discomfort due to the prolonged healing time needed for the donor site.

To avoid this disadvantages of FGG, many alternatives were researched from alternative techniques and methods (i.e., APF), to finding a substitute for the palatal tissue as a donor tissue such as 
the acellular dermal matrix allograft, the platelet-rich fibrin, and the collagen matrix.

The APF was noticed to be clinically useful for a controlled secondary epithelialization appeared ,but it results in high wound contraction and muscle reattachment. APF proved to be significantly better when compared to FGG; especially in regards to morbidity, as patients always preferred it to FGG [17].

The differences between the results of various studies may be related to several factors, including the surgical techniques used in posterior sites only, and using partial-thickness flaps instead than full-thickness flaps to create a periosteal bed for the grafts.

Therefore,the results of this study onincrease of keratinized tissue was not a statistically significant, and By this, we agreed with the study [23, 24], but we disagreed with [5] where the increase was greaterin their studies, and this is often due to the different criteria of inclusion, as it performed the APF during the dental implant process (our study was in the second phase) or due to the difference in the sample size, and we disagreed with [25] and the reason can be attributed to the difference in generation of platelets rich Fibrin, and the follow-up period was less (6 weeks only). As for the results of gingival phenotype in our study, we disagreed with $[26,27]$ and the reason for this increase in their studies (which was not statistically significant in our study) due to the difference in the composition and generation of the platelets rich fibrin and the surgical technique used, and There is also a difference in the high ability of the generations of PRF to synthesize collagen 1, which contributes to increased gingival thickness [28].

Pain is an important adverse effect of periodontal soft-tissue grafting procedures. The VAS scores of pain sensation after FGG in this study are similar to those reported in medical literature [29], and they are significantly higher than those of the (APF+APRF), mainly due to soft-tissue grafting produces causing postoperative discomfort in two areas: the donor site and the recipient site. An advantage of (APF+A-PRF) is the reduction in the number of surgical sites because there is no need for donor tissue. This means that the (APF+A-PRF) technique can be a viable treatment option for patients with concerns about postoperative pain or for those who require multiple bilateral soft-tissue grafts. The majority of shrinkage occurred in the first months after surgery [19]. And the shrinkage when using FGG primarily occurs during the first 28 days. However, after 30 days, no further shrinkage could be seen. So What happens after 3 months is unclear so far.

The shrinkage observed in this study indicates that modifying the surgical procedure may be required for FGG. Urban et al. used strips of FGG in the apical portion of the recipient bed for vestibuloplasty after major bone augmentation [30]. The presence of a strip graft at the apical portion of the recipient bed plays a role as a mechanical barrier against alveolar mucosa and muscle tension, while the A-PRF membrans in this study acts as a scaffold for establishing the KT over periosteum, prevents mucosal relapse, and protects the recipient bed in the vestibular side.

Further clinical studies adding clinical parameters such as plaque index, gingival index, and an evaluation of crestal bone loss should be done to assess the long-term stability of the KT, and its role in maintaining peri-implant health. For these studies, bigger sample sizes are mandatory.

\section{Conclusion}

Within the limitations of this study, it can be concluded that $\mathrm{APF}+\mathrm{A}-\mathrm{PRF}$ can increase the width of the KT. Furthermore, using A-PRF results in decreased postoperative morbidity for the patient in comparison with the FGG. Nevertheless, FGG still is considered the Gold Standard for these types of surgical interventions.

\section{Registration}

The study protocol was registered on ISRCTN registry, with Registration number: ISRCTN33695429; https://doi.org/10.1186/ ISRCTN33695429.

\section{References}

[1]. Tan WL, Wong TL, Wong MC, Lang NP. A systematic review of post-extractional alveolar hard and soft tissue dimensional changes in humans. Clin Oral Implants Res. 2012 Feb;23 Suppl 5:1-21.Pubmed PMID: 22211303.

[2]. Ainamo A, Bergenholtz A, Hugoson A, Ainamo J. Location of the mucogingival junction 18 years after apically repositioned flap surgery. J Clin Periodontol. 1992 Jan;19(1):49-52.Pubmed PMID: 1732309.

[3]. Lim HC, Wiedemeier DB, Hämmerle CH, Thoma DS. The amount of keratinized mucosa may not influence peri-implant health in compliant patients: A retrospective 5-year analysis. J Clin Periodontol. 2019 Mar;46(3):354-62.

[4]. Boynueğri D, Nemli SK, Kasko YA. Significance of keratinized mucosa around dental implants: a prospective comparative study. Clin Oral Implants Res. 2013 Aug;24(8):928-33.Pubmed PMID: 22540356.

[5]. Reddy VK, Parthasarathy H, Lochana P. Evaluating the clinical and esthetic outcome of apically positioned flap technique in augmentation of keratinized gingiva around dental implants. Contemp Clin Dent. 2013 Jul;4(3):319-24. Pubmed PMID: 24124297.

[6]. Newman MG, Takei HH, Carranza FA. Periodontia Clínica. 10 edição. Rio de Janeiro: Sounders Elsevier. 2007:971-83.

[7]. Martins TM, Fernandes LA, Mestrener SR, Saito CT, de Oliveiranobrega FJ, Bosco AF. Apically positioned flap: Reestablishment of esthetics and integrity of the dentogingival unit. Perspect Oral Sci ABR. 2010;2:2175-5124.

[8]. Baltacıoğlu E, Bağış B, Korkmaz FM, Aydın G, Yuva P, Korkmaz YT. Peri-Implant Plastic Surgical Approaches to Increasing Keratinized Mucosa Width. J Oral Implantol. 2015 Jun;41(3):e73-81.Pubmed PMID: 24456001.

[9]. Schmitt CM, Moest T, Lutz R, Wehrhan F, Neukam FW, Schlegel KA. Long-term outcomes after vestibuloplasty with a porcine collagen matrix (Mucograft ${ }^{\bullet}$ ) versus the free gingival graft: a comparative prospective clinical trial. Clin Oral Implants Res. 2016 Nov;27(11):e125-e133.Pubmed PMID: 25720794.

[10]. Ghanaati S, Booms P, Orlowska A, Kubesch A, Lorenz J, Rutkowski J, et al. Advanced platelet-rich fibrin: a new concept for cell-based tissue engineering by means of inflammatory cells. J Oral Implantol. 2014 Dec;40(6):679-89. Pubmed PMID: 24945603.

[11]. Dohan DM, Choukroun J, Diss A, Dohan SL, Dohan AJ, Mouhyi J, et al. Platelet-rich fibrin (PRF): a second-generation platelet concentrate. Part I: technological concepts and evolution. Oral Surg Oral Med Oral Pathol Oral Radiol Endod. 2006 Mar;101(3):e37-44.Pubmed PMID: 16504849.

[12]. Castro AB, Meschi N, Temmerman A, Pinto N, Lambrechts P, Teughels W, et al. Regenerative potential of leucocyte- and platelet-rich fibrin. Part B: sinus floor elevation, alveolar ridge preservation and implant therapy. A systematic review. J Clin Periodontol. 2017 Feb;44(2):225-234.Pubmed PMID: 27891638.

[13]. Kumar YR, Mohanty S, Verma M, Kaur RR, Bhatia P, Kumar VR, et al. Platelet-rich fibrin: the benefits. Br J Oral Maxillofac Surg. 2016 Jan 1;54(1):57-61.

[14]. Kao RT, Curtis DA, Kim DM, Lin GH, Wang CW, Cobb CM, et al. American Academy of Periodontology best evidence consensus statement on modifying periodontal phenotype in preparation for orthodontic and restorative treatment. J Periodontol. 2020 Mar;91(3):289-298.Pubmed PMID: 31943219.

[15]. PAIVA RB, de MENDONÇA JA, ZENÓBIO EG. Peri-implant tissues health and its association to the gingival phenotype. Dent Press Implantol. 2012 Oct $1 ; 6(4)$. 
[16]. Wennström JL, Bengazi F, Lekholm U. The influence of the masticatory mucosa on the peri-implant soft tissue condition. Clin Oral Implants Res. 1994 Mar;5(1):1-8.Pubmed PMID: 8038340.

[17]. Lorenzo R, García V, Orsini M, Martin C, Sanz M. Clinical efficacy of a xenogeneic collagen matrix in augmenting keratinized mucosa around implants: a randomized controlled prospective clinical trial. Clin Oral Implants Res. 2012 Mar;23(3):316-24.Pubmed PMID: 22092380.

[18]. Roccuzzo M, Grasso G, Dalmasso P. Keratinized mucosa around implants in partially edentulous posterior mandible: 10-year results of a prospective comparative study. Clin Oral Implants Res. 2016 Apr;27(4):491-6.Pubmed PMID: 25706508.

[19]. Schmitt CM, Tudor C, Kiener K, Wehrhan F, Schmitt J, Eitner S, et al. Vestibuloplasty: porcine collagen matrix versus free gingival graft: a clinical and histologic study. J Periodontol. 2013 Jul;84(7):914-23.Pubmed PMID: 23030237.

[20]. Misch CE, Al-Shammari KF, Wang HL. Creation of interimplant papillae through a split-finger technique. Implant Dent. 2004 Mar;13(1):20-7.Pubmed PMID: 15017300.

[21]. Urban IA, Nagy K, Werner S, Meyer M. Evaluation of the Combination of Strip Gingival Grafts and a Xenogeneic Collagen Matrix for the Treatment of Severe Mucogingival Defects: A Human Histologic Study. Int J Periodontics Restorative Dent. 2019 Jan/Feb;39(1):9-14.Pubmed PMID: 30543721.

[22]. Metin MU, Dolanmaz DO, Alkan A. Evaluation of autogenous grafts used in vestibuloplasty. J Int Med Res. 2003 Aug;31(4):335-9.

[23]. Thoma DS, Lim HC, Paeng KW, Kim MJ, Jung RE, Hämmerle CHF, et al. Augmentation of keratinized tissue at tooth and implant sites by using autogenous grafts and collagen-based soft-tissue substitutes. J Clin Periodontol. 2020 Jan;47(1):64-71.Pubmed PMID: 31518443.
[24]. Lim HC, An SC, Lee DW. A retrospective comparison of three modalities for vestibuloplasty in the posterior mandible: apically positioned flap only vs. free gingival graft vs. collagen matrix. Clin Oral Investig. 2018 Jun;22(5):2121-2128.Pubmed PMID: 29275490.

[25]. Temmerman A, Cleeren GJ, Castro AB, Teughels W, Quirynen M. L-PRF for increasing the width of keratinized mucosa around implants: A splitmouth, randomized, controlled pilot clinical trial. J Periodontal Res. 2018 Oct;53(5):793-800.Pubmed PMID: 29858875.

[26]. Del Corso M, Sammartino G, Dohan Ehrenfest DM. Letter to the Editor: Re:"Clinical Evaluation of a Modified Coronally Advanced Flap Alone or in Combination With a Platelet-Rich Fibrin Membrane for the Treatment of Adjacent Multiple Gingival Recessions: A 6-Month Study.” J Periodontol. 2009;80(11):1694-7.

[27]. Aroca S, Keglevich T, Barbieri B, Gera I, Etienne D. Clinical evaluation of a modified coronally advanced flap alone or in combination with a plateletrich fibrin membrane for the treatment of adjacent multiple gingival recessions: A 6-month study. J. Periodontol. 2009 Feb;80(2):244-52.

[28]. Burnouf T, Goubran HA, Chen TM, Ou KL, El-Ekiaby M, Radosevic M. Blood-derived biomaterials and platelet growth factors in regenerative medicine. Blood reviews. 2013 Mar 1;27(2):77-89.

[29]. Keceli HG, Aylikci BU, Koseoglu S, Dolgun A. Evaluation of palatal donor site haemostasis and wound healing after free gingival graft surgery. J Clin Periodontol. 2015 Jun;42(6):582-9.Pubmed PMID: 25892528.

[30]. Urban IA, Lozada JL, Nagy K, Sanz M. Treatment of severe mucogingival defects with a combination of strip gingival grafts and a xenogeneic collagen matrix: a prospective case series study. Int J Periodontics Restorative Dent. 2015 May-Jun;35(3):345-53.Pubmed PMID: 25909521. 